\title{
Asteroseismology and interferometry of the red giant star $\epsilon$ Ophiuchi
}

\author{
A. Mazumdar 1,2,3, A. Mérand ${ }^{4,5}$, P. Demarque ${ }^{2}$, P. Kervella $^{6}$, C. Barban ${ }^{6,1}$, F. Baudin ${ }^{7}$, V. Coudé du Foresto ${ }^{6}$, \\ C. Farrington ${ }^{5}$, P. J. Goldfinger ${ }^{5}$, M.-J. Goupil ${ }^{6}$, E. Josselin ${ }^{8}$, R. Kuschnig ${ }^{9}$, H. A. McAlister ${ }^{5}$, J. Matthews ${ }^{10}$, \\ S. T. Ridgway ${ }^{11}$, J. Sturmann ${ }^{5}$, L. Sturmann ${ }^{5}$, T. A. ten Brummelaar ${ }^{5}$, and N. Turner ${ }^{5}$ \\ 1 Instituut voor Sterrenkunde, Katholieke Universiteit, 200D Celestijnenlaan, 3001 Leuven, Belgium \\ e-mail: anwesh@tifr.res.in \\ 2 Astronomy Department, Yale University, PO Box 208101, New Haven CT 06520-8101, USA \\ 3 Homi Bhabha Centre for Science Education, TIFR, V. N. Purav Marg, Mankhurd, Mumbai 400088, India \\ 4 European Southern Observatory, Alonso de Córdova 3107, Casilla 19001, Santiago 19, Chile \\ 5 Center for High Angular Resolution Astronomy, Georgia State University, PO Box 3965, Atlanta, Georgia 30302-3965, USA \\ 6 LESIA, Observatoire de Paris, CNRS UMR 8109, UPMC, Université Paris Diderot, 5 Place Jules Janssen, 92195 Meudon, France \\ 7 Institut d'Astrophysique Spatiale, CNRS/Université Paris XI UMR 8617, 91405 Orsay Cedex, France \\ 8 GRAAL, Université Montpellier II, CNRS UMR 5024, 34095 Montpellier Cedex 05, France \\ 9 Institut für Astronomie, Universität Wien, Türkenschanzstrasse 17, 1180 Vienna, Austria \\ 10 Department of Physics \& Astronomy, University of British Columbia, 6224 Agricultural Road, Vancouver V6T 1Z1, Canada \\ 11 National Optical Astronomy Observatories, 950 North Cherry Avenue, Tucson, AZ 85719, USA
}

Received 20 April 2009 / Accepted 10 June 2009

\section{ABSTRACT}

\begin{abstract}
The GIII red giant star $\epsilon$ Oph has been found to exhibit several modes of oscillation by the MOST mission. We interpret the observed frequencies of oscillation in terms of theoretical radial $p$-mode frequencies of stellar models. Evolutionary models of this star, in both shell H-burning and core He-burning phases of evolution, are constructed using as constraints a combination of measurements from classical ground-based observations (for luminosity, temperature, and chemical composition) and seismic observations from MOST. Radial frequencies of models in either evolutionary phase can reproduce the observed frequency spectrum of $\epsilon$ Oph almost equally well. The best-fit models indicate a mass in the range of $1.85 \pm 0.05 M_{\odot}$ with radius of $10.55 \pm 0.15 R_{\odot}$. We also obtain an independent estimate of the radius of $\epsilon$ Oph with highly accurate interferometric observations in the infrared $K^{\prime}$ band, using the CHARA/FLUOR instrument. The measured limb-darkened disk angular diameter of $\epsilon$ Oph is $2.961 \pm 0.007$ mas. Together with the Hipparcos parallax, this translates into a photospheric radius of $R=10.39 \pm 0.07 R_{\odot}$. The radius obtained from the asteroseismic analysis matches the interferometric value quite closely even though the radius was not constrained during the modelling.
\end{abstract}

Key words. stars: individual: $\epsilon$ Ophiuchi - stars: oscillations - stars: interiors - stars: fundamental parameters techniques: interferometric

\section{Introduction}

Asteroseismology of red giant stars has, in recent years, taken a leap forward with the discovery of pulsations in several Gand K-type giant stars, both from the ground (Frandsen et al. 2002; de Ridder et al. 2006) and from space (Barban et al. 2007; Kallinger et al. 2008b; Hekker et al. 2008). Oscillations in the G giant $\epsilon$ Oph (HD 146791, HR 6075, HIP 79882) were first detected in spectroscopic observations from the ground (de Ridder et al. 2006), although the average large separation could not be distinguished between two possible values because of the daily alias problem. Subsequent observations by the MOST satellite (Walker et al. 2003) led to the discovery of at least 9 radial modes with an average large separation of $5.3 \pm 0.1 \mu \mathrm{Hz}$ (Barban et al. 2007).

This work makes an attempt to interpret the observed frequencies of $\epsilon$ Oph in terms of adiabatic oscillation modes of stellar models in the relevant part of the HR diagram. We constructed red giant models in which the luminosity is provided by either hydrogen burning in a shell outside the helium core, or both shell hydrogen burning and core helium burning. We make quantitative comparisons of these models to the MOST frequencies of $\epsilon$ Oph to determine the stellar parameters like mass, age, radius, and chemical composition. Kallinger et al. (2008a) have earlier presented stellar models in the shell hydrogen-burning phase for $\epsilon$ Oph, based on asteroseismic data. A similar study of oscillations in the red giant $\xi$ Hya in terms of helium burning models was carried out by Christensen-Dalsgaard (2004).

Interferometric measurements of stellar radii are particularly discriminating for models, in particular when combined with asteroseismic frequencies, as noticed, for instance, by Creevey et al. (2007) and Cunha et al. (2007). In this work we report on a new interferometric determination of the radius of $\epsilon \mathrm{Oph}$. While the direct measurement of the radius of a red giant is a useful result in itself, for $\epsilon$ Oph it provides the first opportunity of testing the relevance of theoretical models for red giants that have been calibrated with asteroseismic input. In this study, neither did we use the interferometric radius as an input to the modelling, nor did the interferometric analysis draw upon the asteroseismic information in any way. Thus the radius of the stellar models that 
fit the seismic data best can be tested against the independently measured interferometric radius.

In Sect. 2 we describe the details of the stellar models that we constructed and in Sect. 3 we compare the theoretical frequencies obtained from these models with the observed MOST frequencies. In Sect. 4, we present our new interferometric measurement of the angular diameter of $\epsilon$ Oph. In Sect. 5 we compare the radii of our best seismic models with the interferometric measurement, and discuss our results with similar studies carried out earlier.

\section{Stellar models and theoretical frequencies}

We constructed a grid of stellar models with various input parameters using the Yale Rotating Evolutionary Code (YREC) (Guenther et al. 1992). This code is capable of producing consistent stellar models for low mass giant stars both in the shell $\mathrm{H}$-burning phase and the core He-burning phase (Demarque et al. 2008). We describe these two sets of models below. The radial and nonradial pulsation frequencies of each model are calculated by the oscillation code JIG (Guenther 1994).

\subsection{Input physics}

The models use the latest OPAL equation of state (Rogers \& Nayfonov 2002) and OPAL opacities (Iglesias \& Rogers 1996), supplemented by the low temperature opacities of Ferguson et al. (2005). The nuclear reaction rates from Bahcall \& Pinsonneault (1992) are used. Diffusion of helium and heavy elements have been ignored in the post-main sequence phase of evolution. This is reasonable, since dredge-up by the deep convective envelope present in red giants would mask any effect of diffusion of elements in early phases. The current treatment of convection in the models is through the standard mixing length theory (Böhm-Vitense 1958), which does not properly include the effects of turbulence in the outer layers, and this might substantially affect the frequencies of oscillation (see e.g., Straka et al. 2007). Fortunately, the uncertainty induced in the large separations is much less than in the actual frequencies themselves. Mass loss on the giant branch was not included in the calculations. Most of the mass loss is believed to take place quiescently as the star approaches the tip of the giant branch, as is the case in the commonly adopted Reimers (1977) formulation (Yi et al. 2003). Such mass loss does not affect the thermodynamics of the deep interior appreciably. Most importantly, it takes place at high luminosities, beyond the luminosity of $\epsilon$ Oph on the giant branch. The neutrino losses in the core were taken from the work of Itoh et al. (1989).

\subsection{Range of parameters}

The range of input parameters chosen for the models is dictated by the position of $\epsilon$ Oph on the HR diagram and its estimated chemical composition. The values of effective temperature $\left(\log T_{\text {eff }}=[3.680,3.698]\right)$, luminosity $\left(\log L / L_{\odot}=\right.$ $[1.732,1.806])$, and metallicity $([\mathrm{Fe} / \mathrm{H}]=[-0.07,-0.17])$ are adopted from de Ridder et al. (2006), who have already carried out a detailed survey of these parameters from the literature. Tracks were constructed for different $\left(Y_{0}, Z_{0}\right)$ combinations with $Y_{0}$ ranging from 0.255 to 0.280 , and $Z_{0}$ ranging from 0.012 to 0.015 , corresponding to $[\mathrm{Fe} / \mathrm{H}]=(-0.07,-0.17)$. We adopted the solar abundances as given by Grevesse \& Sauval (1998) in converting $[\mathrm{Fe} / \mathrm{H}]$ to $Z$ values.
For each of these combinations, two values of the mixing length parameter $\alpha$ (the ratio of mixing length to local pressure scale height) have been used: 1.6 and 1.8. Note that using a similar version of YREC and input physics, Kallinger et al. (2008a) used $\alpha=1.74$ in their study of $\epsilon$ Oph. This is the value of $\alpha$ adopted by Yi et al. (2003) for the standard solar model calibration. The radii of red giant models depend sensitively on the choice of $\alpha$.

For each $\left(Y_{0}, Z_{0}, \alpha\right)$, the mass has been varied between 1.8 and $2.4 M_{\odot}$ to check for overlap in the box. In most models, overshoot at the edge of the convective core present on the main sequence was assumed to have negligible effect on the advanced evolution. A few models were constructed with overshoot of 0.2 times the pressure scale height at the convective core edge. Because the size of the convective core is not too large in this mass range, the overall structural effect of core overshoot is modest; but evolutionary timescales are slightly increased when core overshoot is taken into account (Demarque et al. 2004).

\subsection{Shell H-burning models}

Our first set of models for $\epsilon$ Oph are on the ascending red giant branch. These models are characterised by an inert helium core surrounded by a thin hydrogen burning shell. The mass of the shell varies between $0.012 M_{\odot}$ and $0.0007 M_{\odot}$ depending on the mass and age. The mass of the shell decreases as the star ascends the red giant branch. We concentrate on models that lie within the $\epsilon$ Oph box on the HR diagram. For each evolutionary track that traverses the box, several models at slightly different ages are constructed so as to span the box. The theoretical frequencies of these models are compared to the observed frequencies of $\epsilon$ Oph in Sect. 3.

Each evolutionary track was started in the pre-main sequence phase and evolved continuously through core hydrogen burning and eventually shell hydrogen burning along the giant branch. Some of the tracks are plotted in the HR diagram in Fig. 1, together with the $\epsilon$ Oph error box. The size of this box is such that the range of mass of models with a given set of $\left(Y_{0}, Z_{0}\right)$ and $\alpha$ values that pass through the box is $\sim 0.5 M_{\odot}$ (see top panel of Fig. 1). Typically, the mass lies between $1.8 M_{\odot}$ and $2.4 M_{\odot}$, depending on the values of the other parameters. The shift in the tracks with these parameters is also significant (see bottom panel of Fig. 1). Since the outer convective layer of stars in this mass range is extremely thin during the main sequence phase, the tracks with different values of $\alpha$ are almost identical in that phase (e.g., tracks C and D in Fig. 1). But the shift of the track on the giant branch is significant because of the extended convective envelope. In most cases in this study, however, frequencies of models inside the $\epsilon$ Oph box with $\alpha=1.6$ had a poor match with the observed frequencies. Since the tracks move redwards with decreasing $\alpha$, this can be traced to higher mass (and hence larger radii inside the box) of the $\alpha=1.6$ models compared to the $\alpha=1.8$ models. Keeping in mind the adopted range of $[\mathrm{Fe} / \mathrm{H}]$ for the star, the metallicity of the initial model can only be varied between 0.012 and 0.015 with corresponding appropriate change of initial helium abundance between 0.255 and 0.280 to span the entire $\epsilon$ Oph box on the HR diagram.

\subsection{Core He-burning models}

The second set of models are in a later stage of evolution than the first. These models have helium burning in the core of the star. Hydrogen burning in a thin shell outside the core is also 

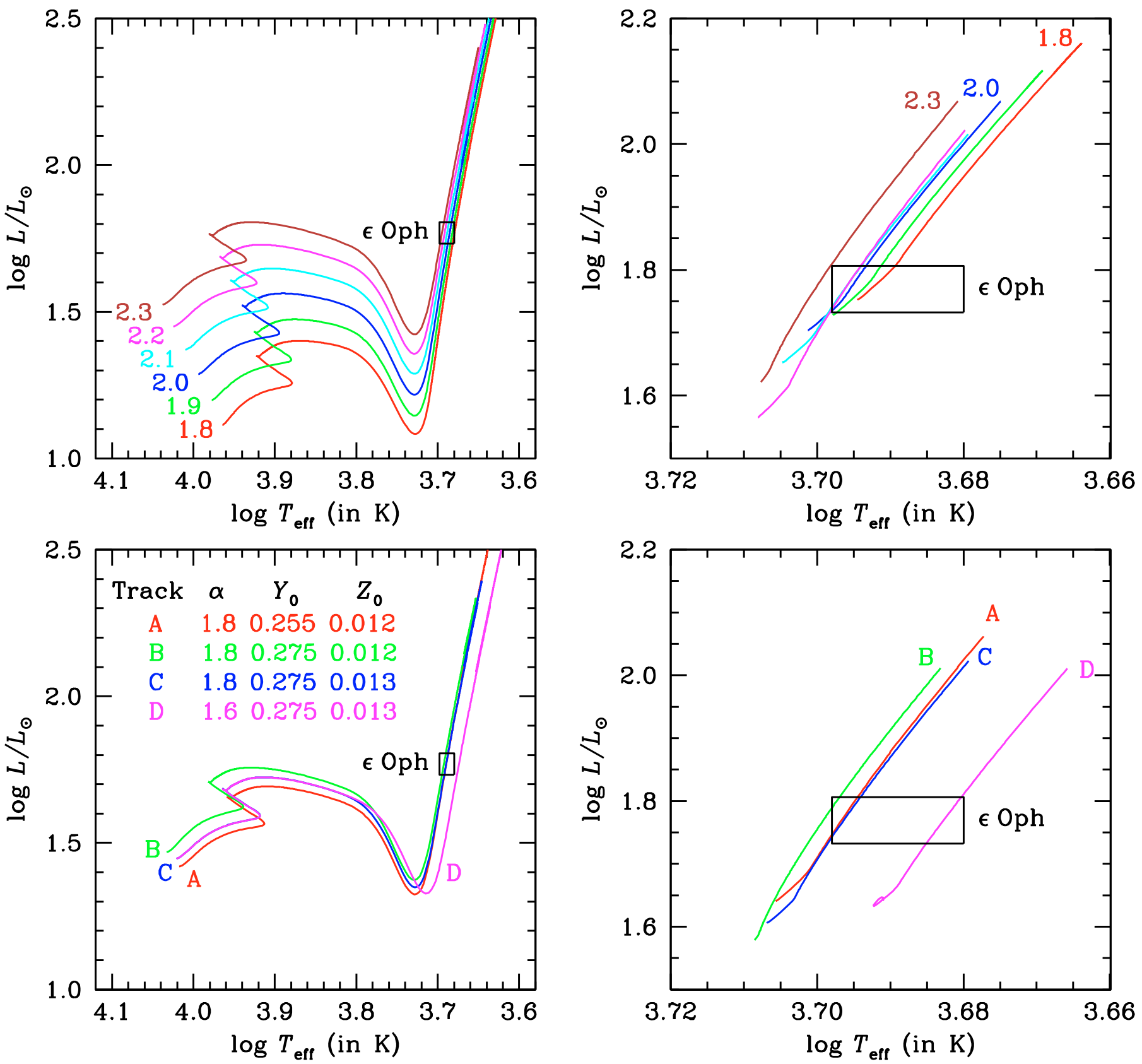

Fig. 1. Stellar evolutionary tracks from the ZAMS to the shell H-burning RGB are shown with respect to the position of $\epsilon$ Oph on the HR diagram. The top panel shows tracks for different masses $\left(M / M_{\odot}\right.$ indicated) with identical initial chemical composition $\left(Y_{0}=0.270\right.$, $\left.Z_{0}=0.012\right)$ and mixing length $(\alpha=1.8)$. The bottom panel shows tracks with the same mass $\left(M=2.2 M_{\odot}\right)$ but with different values of $Y_{0}, Z_{0}$, and $\alpha$.

present. Considering the adopted metallicity value for $\epsilon$ Oph, these models represent the so-called "red clump" stars, rather than metal-poor horizontal branch stars. Indeed, the evolutionary tracks of the models that we constructed lie very close to the red giant branch, and therefore, overlap with the error box of $\epsilon$ Oph on the HR diagram. Figure 2 illustrates these tracks in the HR diagram.

For the range of chemical composition used in our models, it turns out that the helium ignition in the core at the tip of the red giant branch takes place under degenerate conditions for the lower mass stars $\left(M / M_{\odot} \lesssim 2.1\right)$. This is the well-known

Fig. 2. Stellar evolutionary tracks from the ZAHB to the end of He-burning main sequence are shown with respect to the position of $\epsilon$ Oph on the HR diagram. The direction of evolution is from the lower left to the upper right corner of the graphs. The tracks in each panel are the successors of those in Fig. 1. The top panel shows tracks for different masses $\left(M / M_{\odot}\right.$ indicated) with identical values of initial chemical composition and mixing length. The bottom panel shows tracks with the same mass $\left(M=2.2 M_{\odot}\right)$ but with different values of $Y_{0}, Z_{0}$, and $\alpha$ (given in Fig. 1).

"helium flash" mechanism first studied by Schwarzschild \& Härm (1962).

For stars of slightly higher mass $\left(M / M_{\odot} \gtrsim 2.1\right)$, however, the core is not degenerate at the instant helium burning temperatures are reached, and therefore, helium ignition takes place in a controlled fashion. In this latter case, it is numerically easy to continue the evolution of the model past the tip of the red giant branch, and onto the red clump phase. 
For the helium flash scenario, however, the numerical stability of the evolution code at the tip of the giant branch is far less, and only a computationally expensive algorithm involving subtle handling of various parameters can guide the model past the runaway helium ignition process and settle it onto a stable phase of helium burning (Demarque \& Mengel 1971). Piersanti et al. (2004) have demonstrated that even for stars which undergo a violent helium flash, the subsequent evolution of a model on the helium-burning main sequence, i.e., once stable core helium burning has been established, is not very sensitive to the prior history of helium ignition. Specifically, the behaviour of models which have been evolved from appropriate zero age horizontal branch (ZAHB) models with quiescent helium burning in the core is remarkably similar to that of models which have been actually evolved through the helium flash phenomenon. Of course, the make-up of the ZAHB model is crucial - it must reflect the properties of a model that has settled on the helium-burning main sequence after having gone through the helium flash. The critical factor in this starting model, apart from the total mass and the chemical composition at the tip of the giant branch, is the mass of the helium core. Hydrodynamical studies in 2D (Cole \& Deupree 1983) and 3D (Mocàk et al. 2009) confirm this picture, except for possible second order mixing effects due to turbulent overshoot at the convective-radiative interface, which cannot, at this point, be estimated precisely.

For the low mass models we have, therefore, followed the approach of circumventing the numerical difficulties encountered in handling the helium flash, as done by most authors (e.g., Lee \& Demarque 1990; Sweigart 1987). For each mass and chemical composition evolution was continued on the red giant branch till the onset of helium flash. The evolution of the red clump model was then re-started from a ZAHB model of the same total mass with identical chemical composition and helium core mass as the corresponding model at the onset of helium flash at the tip of the red giant branch.

Figure 3 illustrates the behaviour of the central regions of a $2 M_{\odot}$ model near the tip of the giant branch, where helium ignition takes place. The four panels describe the change, as a function of time, or equivalently, as a function of maximum temperature in the star, $T_{\max }$, of the following quantities: the mass contained interior to the shell at $T_{\max }, m\left(T_{\max }\right) / M_{\odot}$, the degeneracy parameter $\eta$, the energy generation rate due to the triplealpha reaction, $\epsilon(3 \alpha)$, and finally the mass of the helium core, $m_{\mathrm{He}-\text { core }} / M_{\odot}$. Note that the shell with the highest temperature is not central (i.e., $m\left(T_{\max }\right) / M_{\odot} \neq 0$ ) and changes with time. This is because of neutrino losses, which are most effective at the higher densities near the centre. As the degeneracy increases, neutrino cooling causes such an inversion of the temperature profile near the centre till the helium ignition temperature is reached. Such off-centre ignition of helium is typical in degenerate helium cores. The energy released in the helium ignition reduces the degeneracy, and the shell of maximum temperature moves back to the centre of the star. The degeneracy parameter $\eta$ is a measure of the degree of degeneracy of the electron gas. It is a dimensionless parameter used to quantify the relationship between the electron density and pressure in a partially degenerate electron gas. The detailed formalism used in the models is that described by Clayton (1968, p. 64). We note, while inspecting Fig. 3, that the quantity $\eta$ varies between $-\infty$ in the ideal gas case and $+\infty$ in a fully degenerate Fermi-Dirac gas. The mass of the helium core keeps increasing throughout the red giant phase because of hydrogen burning in the shell immediately above it till the onset of helium burning. The maximum mass of a degenerate helium core at helium flash is typically $\sim 0.45 M_{\odot}$,

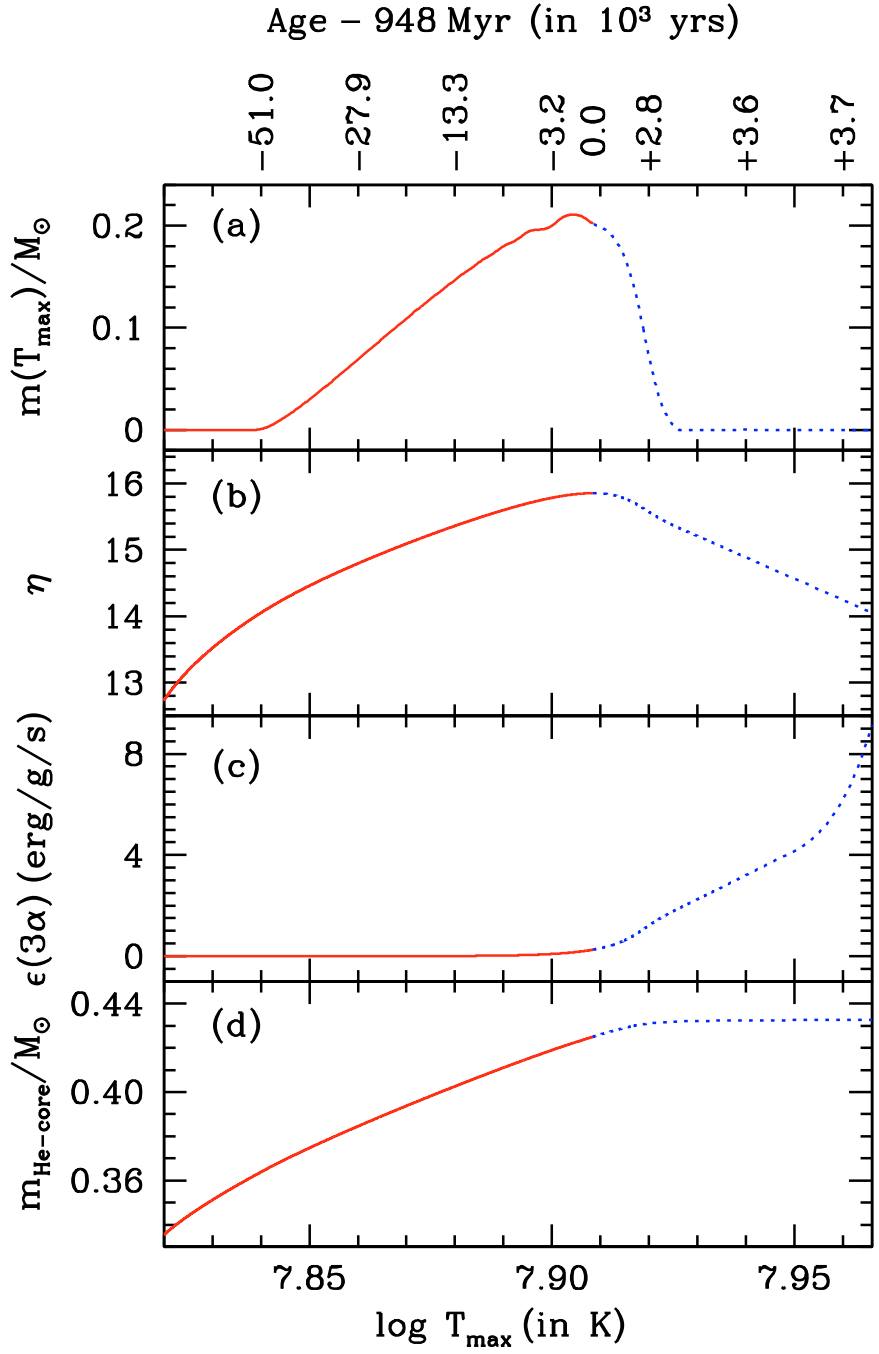

Fig. 3. The change, as a function of time (upper $x$-axis), or equivalently as a function of maximum temperature in the star, $T_{\max }$ (lower $x$-axis), of a) the mass interior to the shell at $T_{\max }, m\left(T_{\max }\right) / M_{\odot}$; b) the degeneracy parameter, $\eta$; c) the energy generation due to the triple-alpha reaction, $\epsilon(3 \alpha)$; and $\mathbf{d})$ the mass of the helium core, $m_{\mathrm{He}-\text { core }} / M_{\odot}$, close to the onset of He-burning of a $2 M_{\odot}$ star are shown. The red solid line denotes the pre-He-ignition phase, while the blue dotted line shows the post-He-ignition phase.

irrespective of the total mass of the star, and depends slightly on the other stellar parameters.

The actual age of the model on the helium-burning main sequence cannot, of course, be assigned accurately because of the "missing" period of the helium flash. However, the total duration of the helium flash phenomenon and the subsequent stabilisation of the star on the helium-burning main sequence is only $\sim 1.5$ Myr (Piersanti et al. 2004), and hence the uncertainty in the age in our helium-burning models is quite small.

For slightly higher mass models $\left(M / M_{\odot} \gtrsim 2.2\right)$, the evolution of the star is followed continuously from the pre-main sequence stage till the red clump stage. Since the helium ignition at the tip of the giant branch occurs under non-degenerate conditions, there are no numerical problems in such cases. The transition mass from violent to quiescent helium ignition is a function of chemical composition. It has been studied in detail by Sweigart et al. (1989). More recent illustration is found in the 

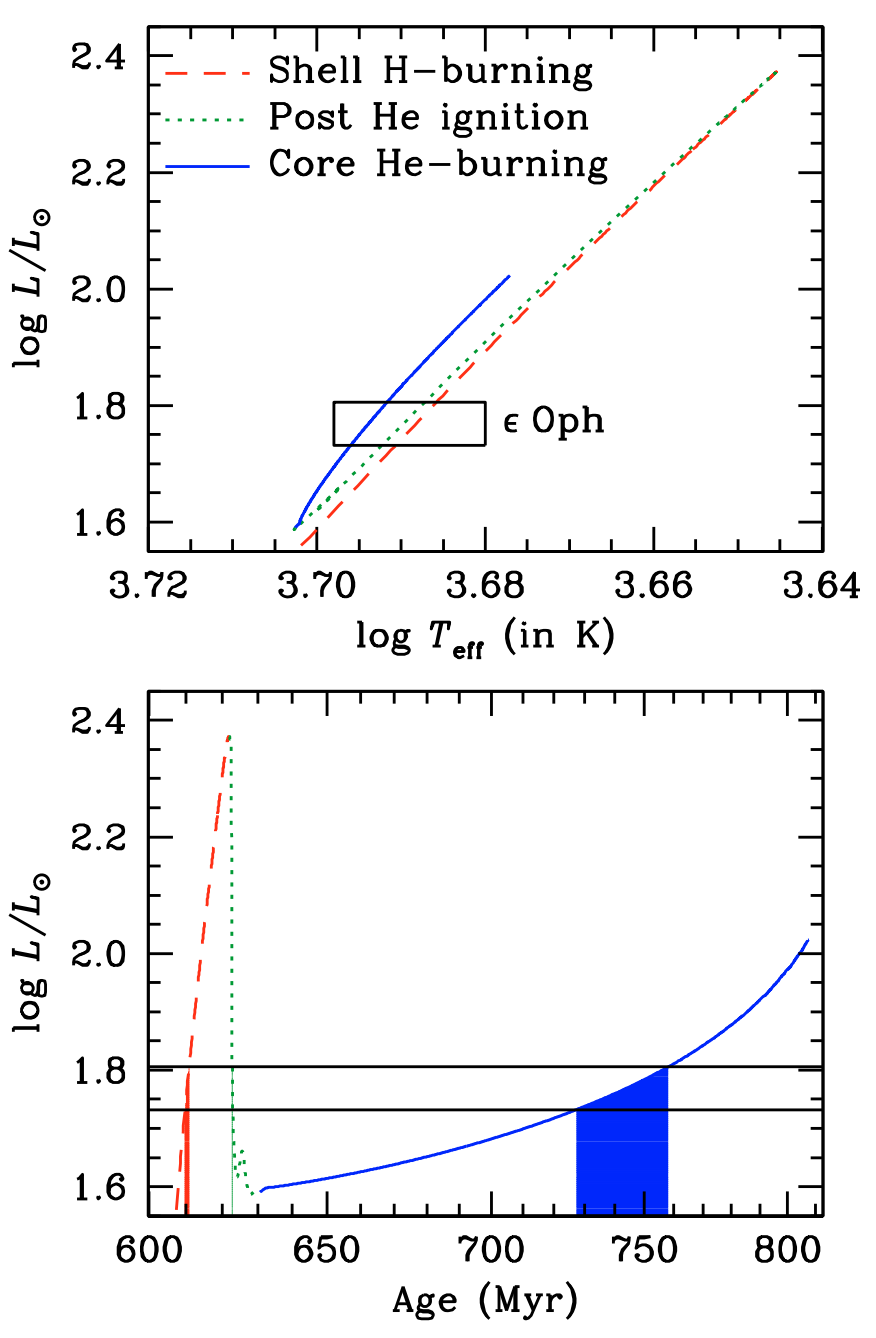

Fig. 4. The different timescales of the stellar evolutionary tracks in each crossing of the $\epsilon$ Oph box for the shell H-burning, post He-ignition, and core He-burning main sequence are shown. In the top panel the full evolutionary track of a $2.3 M_{\odot}$ model is shown on the HR diagram. The bottom panel shows the luminosity of the star as a function of its age. The luminosity limits of $\epsilon$ Oph are indicated by the two horizontal lines, and the time spent by the star within these limits in each phase are indicated by the projections on the $x$-axis.

tracks of Yi et al. (2003), which were constructed with a similar version of YREC (Demarque et al. 2008).

There is an uncertainty in the age of the core helium-burning models because of the assumption of no mass loss on the red giant branch. Since the amount of mass lost in the giant phase is not known, the mass of the ZAHB model cannot be assigned accurately. Therefore, the ages of helium-burning models given in Tables 1 and 4 are, in fact, upper limits to the real ages.

\subsection{Timescales of evolution}

A star of a given mass might cross the $\epsilon$ Oph errorbox on the HR diagram three times - once upwards during shell H-burning, once downwards during the stabilisation of the star just after He ignition, and once again upwards during the phase of stable core He-burning. The timescales of evolution in these three phases are quite different, and consequently the time spent inside the $\epsilon$ Oph box differs vastly. Figure 4 illustrates the timescales of evolution in each crossing of the box for a $2.3 M_{\odot}$ star. During the shell H-burning phase, it spends nearly 1.09 Myr inside the box. After He ignition in the core, it spends only 0.17 Myr during the rapid settling towards the He-burning main sequence, and finally it spends $30.59 \mathrm{Myr}$ during the stable core He-burning phase. Similar timescales are found for other stars in this mass range. The time spent during the shell H-burning phase is typically $\sim 20$ times shorter than that during the core He-burning phase. Thus the probabilities of $\epsilon$ Oph being in the corresponding phases of evolution are in the same ratio.

\section{Comparison of theoretical and observed frequencies}

The theoretical frequencies of the stellar models were compared with the MOST data on $\epsilon$ Oph. Typically, in asteroseismic modelling studies, the comparison between a stellar model and the observed frequency data is carried out in terms of frequency separations, especially the large frequency separations, rather than the frequencies themselves. This is done to eliminate the uncertainty in the theoretical absolute frequencies due to inadequate modelling of the stellar surface layers. However, this is possible only in the happy circumstance of detection of a series of frequencies of the same degree and successive radial orders, for which the large separations can be determined. For $\epsilon$ Oph, Barban et al. (2007) indeed provide the frequencies of 9 successive radial order modes. Thus it is possible to match the observed large separations with the theoretical values from the models. However, as an additional comparison, we also match the absolute frequencies of radial modes of our stellar models with the MOST data.

For each comparison, a reduced $\chi^{2}$ value is computed as

$\chi_{v}^{2}=\frac{1}{N} \sum_{i=1}^{N}\left[\frac{v_{\mathrm{MOST}}-v_{\text {model }}}{\delta v_{\mathrm{MOST}}}\right]^{2}$

$\chi_{\Delta v}^{2}=\frac{1}{N-1} \sum_{i=2}^{N}\left[\frac{\Delta v_{\mathrm{MOST}}-\Delta v_{\text {model }}}{\delta \Delta v_{\mathrm{MOST}}}\right]^{2}$

where $v$ and $\Delta v$ represent the frequency and large separation respectively, and $N$ is the number of observed modes compared (maximum of 9). $\delta v_{\text {MOST }}$ is the measured error in the MOST frequency and $\delta \Delta v_{\text {MOST }}$ is the error in the large separation computed by adding the adjacent frequency errors in quadrature. Thus for each model, values of $\chi_{v}^{2}$ and $\chi_{\Delta v}^{2}$ can be computed. A small value of $\chi_{v}^{2}$ would usually imply a small value for $\chi_{\Delta v}^{2}$, but the reverse is not necessarily true. It is possible to find models for which the large separations are quite close to the observed values, but all the frequencies are shifted by a nearly constant amount. Given the inadequacies of input physics in the models, especially in the rarefied outer convective layers, the absolute values of the theoretical frequencies cannot be trusted too much. The large separations, on the other hand, would be relatively free from such ambiguities, and would reflect the overall structure of the star better. This is why we put a greater importance on minimising $\chi_{\Delta v}^{2}$, rather than $\chi_{v}^{2}$, in choosing our best model for $\epsilon$ Oph. It turns out, however, that the models with lowest $\chi_{\Delta v}^{2}$ have reasonably small values for $\chi_{v}^{2}$ as well in most cases. Kallinger et al. (2008a) have used absolute frequencies in their comparison of models and observations of $\epsilon$ Oph.

The best-fitting models in both shell H-burning and core He-burning phases can reproduce the observed large separations fairly well. This is illustrated in Fig. 5. However, it turns out that 
Table 1. Stellar parameters for the models with lowest $\chi_{\Delta v}^{2}$ and $\chi_{v}^{2}$ values in either shell H-burning or core He-burning phase.

\begin{tabular}{clcccccccccc}
\hline \hline Minimised by & Evolutionary phase & $Y_{0}$ & $Z_{0}$ & $\alpha$ & $M / M_{\odot}$ & Age (Myr) & $\log T_{\text {eff }}$ & $\log L / L_{\odot}$ & $R / R_{\odot}$ & $\chi_{v}^{2}$ & $\chi_{\Delta v}^{2}$ \\
\hline \multirow{2}{*}{$\chi_{\Delta v}^{2}$} & Shell H-burning & 0.260 & 0.012 & 1.8 & 1.9 & 1092 & 3.682 & 1.732 & 10.54 & 10.68 & 0.62 \\
\multirow{2}{*}{$\chi_{v}^{2}$} & Core He-burning & 0.265 & 0.012 & 1.8 & 1.8 & 1326 & 3.692 & 1.766 & 10.51 & 0.55 & 0.59 \\
& Shell H-burning & 0.260 & 0.013 & 1.8 & 1.9 & 1063 & 3.681 & 1.741 & 10.71 & 0.47 & 0.63 \\
& Core He-burning & 0.255 & 0.012 & 1.8 & 1.9 & 1173 & 3.692 & 1.780 & 10.69 & 0.51 & 0.68 \\
\hline
\end{tabular}

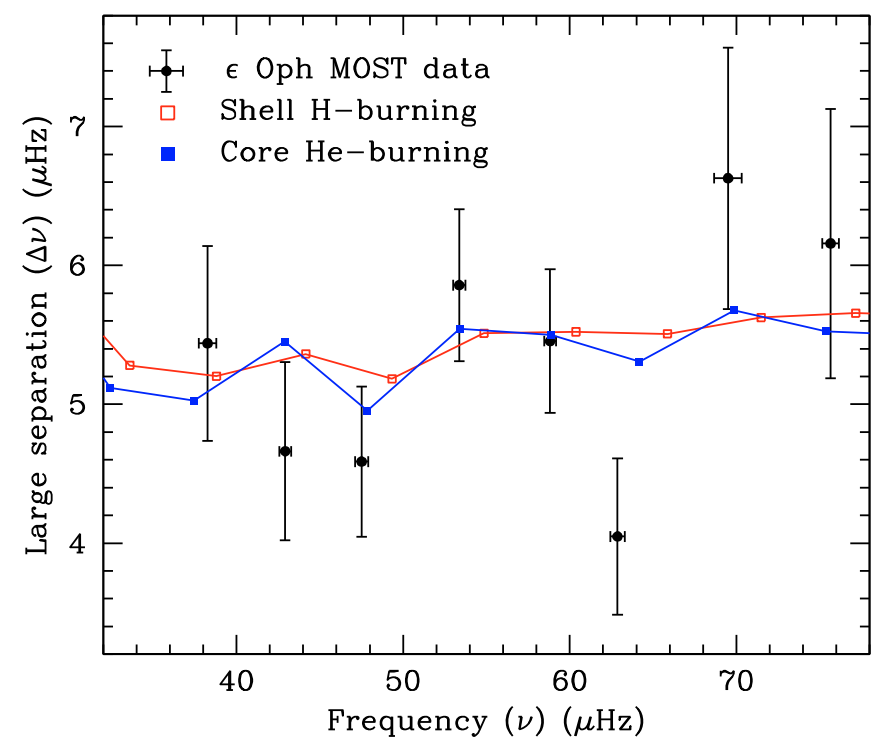

Fig. 5. The large frequency separations of best-fitting radial modes of stellar models in the shell H-burning (red open squares) and core Heburning (blue filled squares) phases inside the $\epsilon$ Oph errorbox are shown in comparison with the observed separations of $\epsilon$ Oph. The parameters of these models are given in Table 1. These models are chosen on the basis of lowest $\chi_{\Delta v}^{2}$ criterion.

the large separation corresponding to one of the observed modes at $\nu_{\text {MOST }}=62.871 \mu \mathrm{Hz}$ is nearly $2 \sigma$ away from the theoretical value in all our models. This data point is always the largest contributor to $\chi_{v}^{2}$ and $\chi_{\Delta v}^{2}$. We have ignored this point while choosing our best model.

The parameters of the models with the lowest $\chi_{\Delta v}^{2}$ and $\chi_{v}^{2}$ values are listed in Table 1 . Notice that the model with least $\chi_{\Delta v}^{2}$ in the shell $\mathrm{H}$-burning phase has a significantly high value of $\chi_{v}^{2}$ because of an overall shift in the absolute frequencies. Given one criterion for comparison (either $\chi_{\Delta v}^{2}$ or $\chi_{v}^{2}$ ), it is clear that the models in the shell H-burning phase fit the data almost equally well as those in the core He-burning phase. Thus, the present data is unable to distinguish between these two phases of stellar evolution. However, as discussed in Sect. 2.5, the likelihood of the star being in the core He-burning phase is greater than it being in the shell H-burning phase.

Based on the best match between observed and model large separations, the models indicate very similar parameters for both phases of evolution. We estimate the stellar parameters from not only the models with lowest $\chi^{2}$, but actually all models that have $\chi^{2}$ values within $50 \%$ of the least $\chi^{2}$. The mass is estimated to be $1.85 \pm 0.05 M_{\odot}$, while the metallicity of the best models are in the range of $0.0125 \pm 0.0005$. The radius lies in the range of $10.55 \pm 0.15 R_{\odot}$. The radius of $\epsilon \mathrm{Oph}$, however, can be measured independently through interferometry, as described in the next section.
Table 2. Calibrators used for $\epsilon$ Oph.

\begin{tabular}{lccccc}
\hline \hline Star & $m_{V}$ & $m_{K \mathrm{~s}}$ & Sp. Type & $\theta_{\mathrm{LD}}(\mathrm{mas})$ & $\gamma\left({ }^{\circ}\right)$ \\
\hline HR 145085 & 5.9 & 2.4 & K5III & $1.677 \pm 0.022$ & 8 \\
HD 162468 & 6.2 & 3.2 & K1III & $1.154 \pm 0.015$ & 28 \\
HD 166460 & 5.5 & 2.6 & K2III & $1.439 \pm 0.018$ & 29 \\
\hline
\end{tabular}

Table 3. Squared visibility measurements obtained for $\epsilon$ Oph.

\begin{tabular}{lrrc}
\hline \hline MJD & $B(\mathrm{~m})$ & $\mathrm{PA}\left(^{\circ}\right)$ & $V^{2} \pm \sigma\left(V^{2}\right)$ \\
\hline 53936.21641 & 154.375 & -32.471 & $0.03131 \pm 0.00173$ \\
53936.25567 & 165.132 & -36.878 & $0.01450 \pm 0.00049$ \\
53937.20561 & 151.978 & -31.267 & $0.03746 \pm 0.00104$ \\
53937.23681 & 160.961 & -35.346 & $0.01901 \pm 0.00055$ \\
53937.26478 & 167.893 & -37.774 & $0.01054 \pm 0.00028$ \\
\hline
\end{tabular}

\section{Interferometric measurements}

\subsection{Instrumental setup}

We observed $\epsilon$ Oph in July 2006 at the CHARA Array (ten Brummelaar 2005) using FLUOR, the Fiber Linked Unit for Optical Recombination (Coudé du Foresto et al. 2003). This instrument is equipped with a near infrared $K^{\prime}$ band filter $(1.9 \leq$ $\lambda \leq 2.35 \mu \mathrm{m}$ ). We extracted the instrumental visibilities from the raw data using the FLUOR data reduction software (Coudé du Foresto et al. 1997; Kervella et al. 2004; Mérand 2006). For all the reported observations, we used the CHARA baselines $\mathrm{S} 2$ W2, with ground lengths of $177 \mathrm{~m}$, which is mostly a northsouth baseline in orientation. The calibrator stars were chosen in the catalogue compiled by Mérand et al. (2005), using criteria defined by these authors. They were observed immediately before or after our targets in order to monitor the interferometric transfer function of the instrument. These are listed in Table 2 where the limb darkened angular diameter, $\theta_{\mathrm{LD}}$, and the angular separation, $\gamma$, with $\epsilon$ Oph is given for each calibrator. For a more detailed description of the observing procedure and the error propagation, the interested reader is referred to Kervella et al. (2008) and Perrin (2003), respectively. The resulting calibrated squared visibilities are listed in Table 3 , where $B$ is the projected baseline length, and "PA" is the azimuth of the projected baseline (counted positively from North to East).

\subsection{Angular diameter measurement and precision}

In order to accurately measure $\epsilon$ Oph angular diameters, we used many known stellar calibrators and we repeated the observation on two separate and consecutive nights. Since, in the end, the number of visibility will always be small, statistically speaking, the final confidence on the precision will rely more on the repeatability of the the result and the consistency between the stellar calibrators. 
Our observation strategy was designed to give maximum precision and confidence to our results. As a result, we achieve:

- the repeatability of the result. The first night gives $\theta_{\mathrm{UD}}=$ $2.881 \pm 0.006$ mas, with a reduced $\chi^{2}$ of 0.9 ; the second night $\theta_{\mathrm{UD}}=2.891 \pm 0.005$ mas, with a reduced $\chi^{2}$ of 0.3 . This is consistent at the 0.01 mas level.

- the use of multiple slightly resolved calibrators of various sizes. Indeed, if we assume we have an overall bias in the angular diameter estimation of the calibrators, it is going to lead to a differential calibrated visibility bias, depending on the size of the calibrator. For example, if we multiply the diameters of our calibrators by 1.05 (i.e. a $5 \%$ bias), the new diameter for $\epsilon$ Oph is $\theta_{\mathrm{UD}}=2.895 \pm 0.005$ mas, with a reduced $\chi^{2}$ of 2.5 instead of our result $\theta_{\mathrm{UD}}=2.888 \pm 0.003$ mas, with a reduced $\chi^{2}$ of 1.0. Not only would our final result be barely affected, but also the reduced $\chi^{2}$ becomes much higher, because points calibrated by our large calibrator become completely inconsistent with the rest of the batch.

Another possible source of bias, and possibly the ultimate one, is the wavelength calibration. This has been done by Mérand (2005) and the process leads to a wavelength calibration accuracy of $0.005 \mu \mathrm{m}$. We changed the software wavelength calibration by this amount and redid the reduction and calibration. This led to a bias of 0.006 mas. Hence, we added quadratically a 0.006 mas error, leading to a final uncertainty of 0.007 mas.

\subsection{Limb darkened angular diameter and photospheric radius}

In order to estimate the unbiased angular diameter from the measured visibilities it is necessary to know the intensity distribution of the light on the stellar disk, i.e., the limb darkening (LD). As we do not fully resolve $\epsilon$ Oph, we cannot measure the limb darkening directly from the data. We thus model it using the MARCS models (Gustafsson et al. 2008) ${ }^{1}$ for the computation of the intensity profile of the star, taking into account the actual spectral transmission function of the FLUOR instrument (Mérand 2005). The LD coefficients have been computed with the TURBOSPECTRUM code (Alvarez \& Plez 1998). The result is shown in Fig. 6. It is to be noted that taking an intensity profile from a different model, say the one predicted by the ATLAS9 model from Kurucz, using Claret's laws (Claret 2000), results in the same final result, within fractions of the statistical error bar. The reason is that for the relatively large spectral bandwidth of FLUOR and considering that we measure firstlobe visibilities only, the difference between the MARCS and ATLAS models is negligible (of the order of a fraction of our diameter error bar). The magnitude of the limb darkening effect being much smaller in the infrared than in the visible, our resulting limb darkened angular diameter measurement in the $K$ band is largely unaffected by the choice of the limb darkening model.

The result of the visibility fit is presented in Fig. 7 using the MARCS limb darkening model. We derive the following limb darkened disk angular diameter:

$\theta_{\mathrm{LD}}(\epsilon \mathrm{Oph})=2.961 \pm 0.003$ (stat.) \pm 0.006 (syst.) mas.

This value is compatible with the spectrophotometric angular diameter estimate by Cohen et al. (1999) of $\theta_{\mathrm{LD}}=3.00 \pm 0.03$ mas. We took $\epsilon$ Oph's parallax from the reprocessed Hipparcos catalogue by van Leeuwen (2007a,b):

$\pi(\epsilon \mathrm{Oph})=30.64 \pm 0.20$ mas $( \pm 0.65 \%)$.

1 http://www.marcs.astro.uu.se/

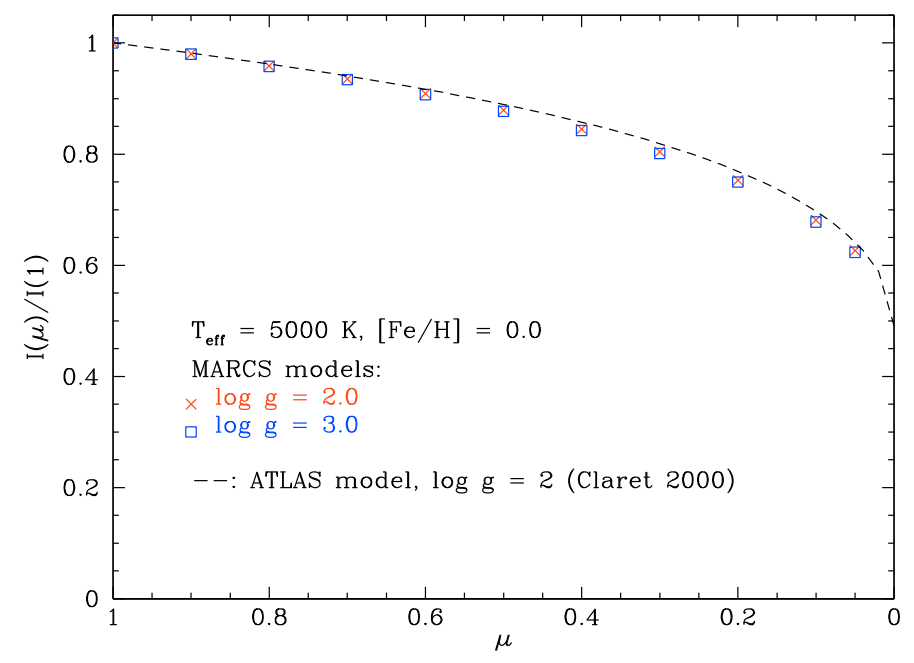

Fig. 6. Comparison of the intensity profiles of $\epsilon$ Oph from the MARCS (red crosses and blue squares for two values of $\log g$ ) and Claret's (Claret 2000) (dashed curve) model.

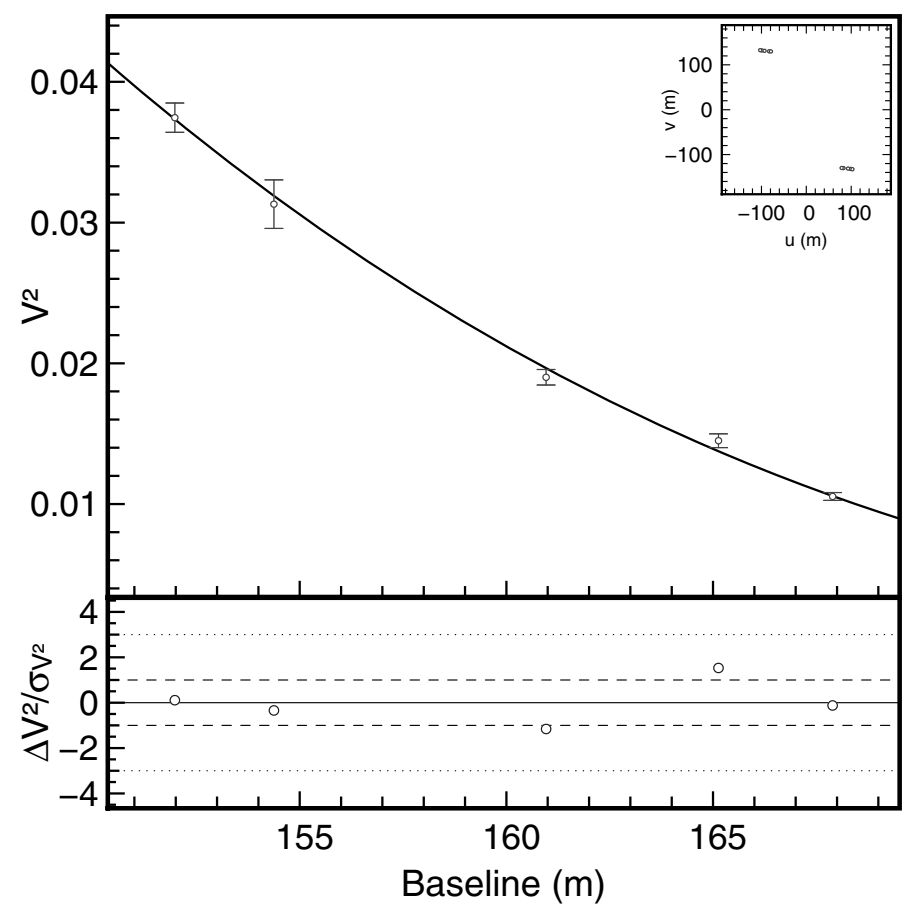

Fig. 7. Squared visibilities and adjusted limb darkened disk visibility model for $\epsilon$ Oph (see also Table 3). The lower panel shows the residuals to the model, with $1 \sigma$ (dashed line) and $3 \sigma$ (dotted line) limits. The inset panel shows the $(u, v)$ coverage. Note that the error bar given here is the formal error bar: we added some systematics for final result (see text).

This value compares well with van Altena et al. (1995), and the original Hipparcos catalogue (ESA 1997), but is more precise. We finally derive the photospheric linear radius:

$R(\epsilon \mathrm{Oph})=10.39 \pm 0.07 R_{\odot}( \pm 0.67 \%)$.

In spite of the the relatively high precision of the parallax, it is by far the limiting factor for the precision of the radius. 


\section{Discussion}

In this study we have constructed stellar models of red giants in both shell H-burning and core He-burning phases and compared their adiabatic frequencies with the frequencies of $\epsilon$ Oph observed by the MOST satellite and published by Barban et al. (2007). We have also measured the radius of the star through optical interferometry using the CHARA/FLUOR instrument.

We have demonstrated that the observed frequencies of $\epsilon$ Oph are consistent with radial $p$-mode pulsations of a red giant at the relevant position on the HR diagram. Unfortunately, the radial mode frequencies cannot distinguish between the two phases of stellar evolution - shell H-burning and core Heburning. This is hardly surprising, since the models inside the box on the HR diagram in either phase would have approximately similar radii, and the large separation depends crucially on the radius of the star. However, the seismic information helps us to constrain the radius of the star to a much narrower range than that possible by the errorbox on the HR diagram.

Kallinger et al. (2008a) have carried out a seismic modelling study of $\epsilon$ Oph also, but with important differences. Firstly, they have interpreted the observed peaks in the power spectrum of $\epsilon$ Oph to be radial as well as nonradial modes. They identify the sharp narrow peaks in the power spectrum as long-lived nonradial modes, as compared to the broad Lorentzian envelopes, resulting in short-lived modes, which have been identified as radial modes by Barban et al. (2007). Secondly, they have matched the observed frequency values, and not the large separations, to the theoretical model frequencies. Lastly, they have used only shell H-burning models. Given these differences, it is not surprising that they obtained a slightly different result than ours.

Little is known whether $p$-modes, radial or nonradial, can be excited in red giants to an amplitude high enough to be observable. A detailed theoretical study of models of $\alpha \mathrm{UMa}$, observed by Buzasi et al. (2000) with the WIRE satellite, and of similar $2 M_{\odot}$ models on the lower giant branch, by Dziembowski et al. (2001) provides some insight on the oscillation characteristics of lower giant branch stars. Giant stars are characterised by an inner cavity that can support gravity waves ( $g$-modes), and an outer cavity that supports acoustic waves ( $p$-modes). Observable modes in the outer cavity are mixed modes, with a $g$-mode character in the inner cavity and $p$-mode character in the outer cavity. Dziembowski et al. (2001) showed that such mixed $p$-modes can have substantial amplitudes, and that low degree modes with $\ell=2,3$, together with the radial $p$-modes can be unstable. According to their models of lower giant branch stars, high amplitudes in the outer cavity arise only for modes with $\ell=2$. The excitation properties of $p$-modes in more luminous red giants lying around the middle of the red giant branch, which have very deep convection zones, such as $\epsilon$ Oph, are mostly unexplored.

A careful visual examination of the observed frequency spectrum of $\epsilon$ Oph (Barban et al. 2007) reveals a clear comblike structure with reasonably regular spacing of $\sim 5.3 \mu \mathrm{Hz}$. In an adiabatic pulsation calculation of a theoretical model, a series of regularly spaced radial modes accompanied by a dense forest of nonradial modes are obtained (see Fig. 8). The observed frequencies of $\epsilon \mathrm{Oph}$, as given by Barban et al. (2007), can be matched reasonably well to the radial modes. In principle, they can also be matched easily to many of the closely spaced nonradial modes as well, but the question remains as to why the majority of the nonradial modes are not observed.

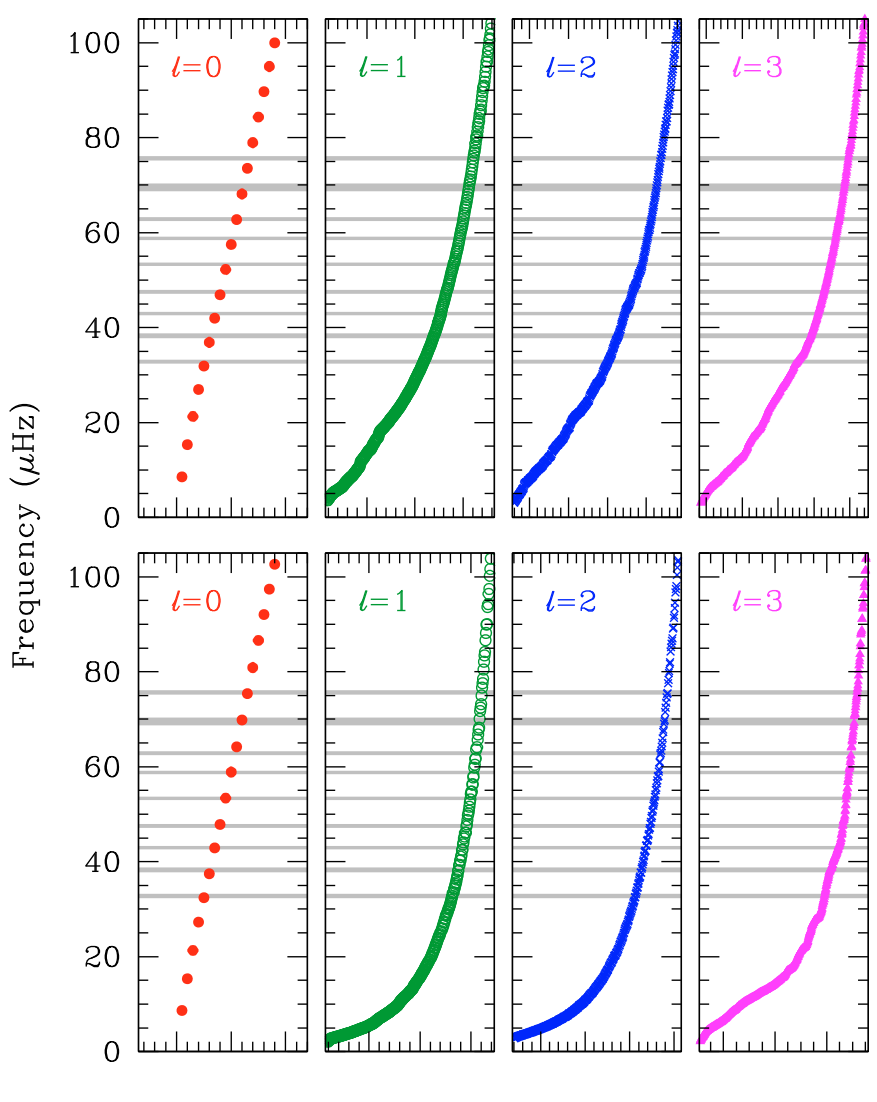

Arbitrary radial order

Fig. 8. Adiabatic pulsation frequencies of radial $(\ell=0)$ and nonradial $(\ell=1,2,3)$ modes in a typical red giant model inside the $\epsilon$ Oph errorbox on the HR diagram are shown. The frequencies of each degree are plotted in increasing order of magnitude against some arbitrary assignment of radial order (which can be simply treated as the serial number of the mode in the eigenspectrum). The top row shows the frequencies of a $1.8 M_{\odot}$ star inside the $\epsilon$ Oph in the shell H-burning phase. The bottom row shows the frequencies of the same star when it again appears inside the $\epsilon$ Oph errorbox in the later phase of stable core He-burning. The horizontal grey bands represent the observed MOST frequencies of $\epsilon$ Oph with $\pm 1 \sigma$ error bars.

A possible explanation of this may be provided in terms of the normalised mode inertia $E$, defined according to Christensen-Dalsgaard (2004) as

$E=\frac{\int_{V} \rho|\xi(r)|^{2} \mathrm{~d} V}{M|\xi(R)|^{2}}$

where $\boldsymbol{\xi}$ is the displacement vector, and the integration is carried out over the volume $V$ of the star. Most nonradial modes are strongly trapped in the core, with only a few modes being trapped in the envelope. The modes trapped in the core have high inertia and therefore unlikely to have enough surface amplitudes. On the other hand, the modes trapped in the envelope can have inertia as low as the radial modes, and therefore, be seen on the surface. Figure 9 shows the normalised mode inertia for radial as well as nonradial modes in a typical model inside the $\epsilon$ Oph errorbox. Only selected $\ell=1,2,3$ modes have inertia comparable to the radial modes. However, a reliable estimate of the surface amplitude can only be obtained when nonadiabatic effects in the outer layers are taken into account.

In a recent theoretical work involving nonadiabatic treatment of the excitation mechanism, Dupret et al. (2009) have found that 


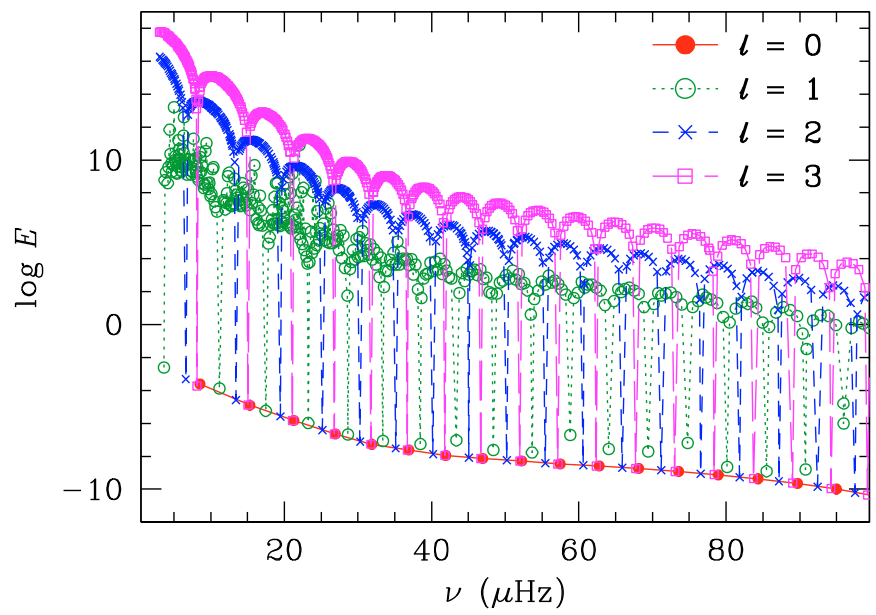

Fig. 9. Normalised mode inertia of $\ell=0,1,2,3$ modes as a function of frequency is shown for a $1.8 M_{\odot}$ star in shell hydrogen burning phase inside the $\epsilon$ Oph errorbox. The individual eigenfrequencies are joined by lines for each degree (red filled circles with solid lines for $\ell=0$; green empty circles with dotted lines for $\ell=1$; blue crosses with short dashed lines for $\ell=2$; magenta empty squares with long dashed lines for $\ell=3$ ).

despite their low amplitudes, a selection of nonradial modes may still have appreciable heights in the power spectrum of red giants because of their long lifetimes, and thus be detected in observations. However, the detection of such modes depends crucially on the evolutionary stage of the star on the red giant branch. Theoretical computations for intermediate red giant branch stars like $\epsilon$ Oph predict much longer lifetimes for nonradial modes ( $~ \$ 50$ days) than radial modes ( $\$ 20$ days) because of their larger inertia. But if the duration of observation is shorter than these lifetimes, as the case is for MOST observations of $\epsilon \mathrm{Oph}$, it is not possible to resolve these nonradial modes in the power spectrum. This implies that the modes attain smaller heights in the power spectrum and they become extremely difficult to detect.

Further, in the higher part of the frequency domain, the detectable nonradial modes also appear with an asymptotic regular separation pattern similar to that found in main sequence stars. This means that the $\ell=0$ and $\ell=2$ modes will appear close to each other, with the $\ell=1$ modes occurring roughly midway between them. For $\epsilon$ Oph such a pattern implies that if we consider nonradial modes to be present in the spectrum, the large separation (of $\ell=0$ modes, for example) would be almost double the value than that obtained by postulating only radial modes. Such a high value of the large separation $(\sim 11 \mu \mathrm{Hz})$ is inconsistent with the position of $\epsilon \mathrm{Oph}$ on the HR diagram. However, according to Dupret et al. (2009), the lifetimes of some $\ell=2$ modes which are strongly trapped in the envelope are comparable to that of the radial ones. So these envelope-trapped $\ell=2$ modes could indeed be detectable. Trapping of $\ell=1$ modes is less efficient, as found by Dziembowski et al. (2001) too, and hence they may not be observable. However, in the absence of any detailed calculations for the mode amplitudes and lifetimes for the specific case of $\epsilon \mathrm{Oph}$, in this work we have adopted Barban et al. (2007)'s interpretation of the frequencies as radial modes only. An alternative modelling analysis taking into account the possibility of nonradial modes might lead to a different set of model parameters, as found by Kallinger et al. (2008a), for example. The theoretical justification behind the presence of only a few specific nonradial modes among the possible dense spectrum of such modes requires further detailed study.

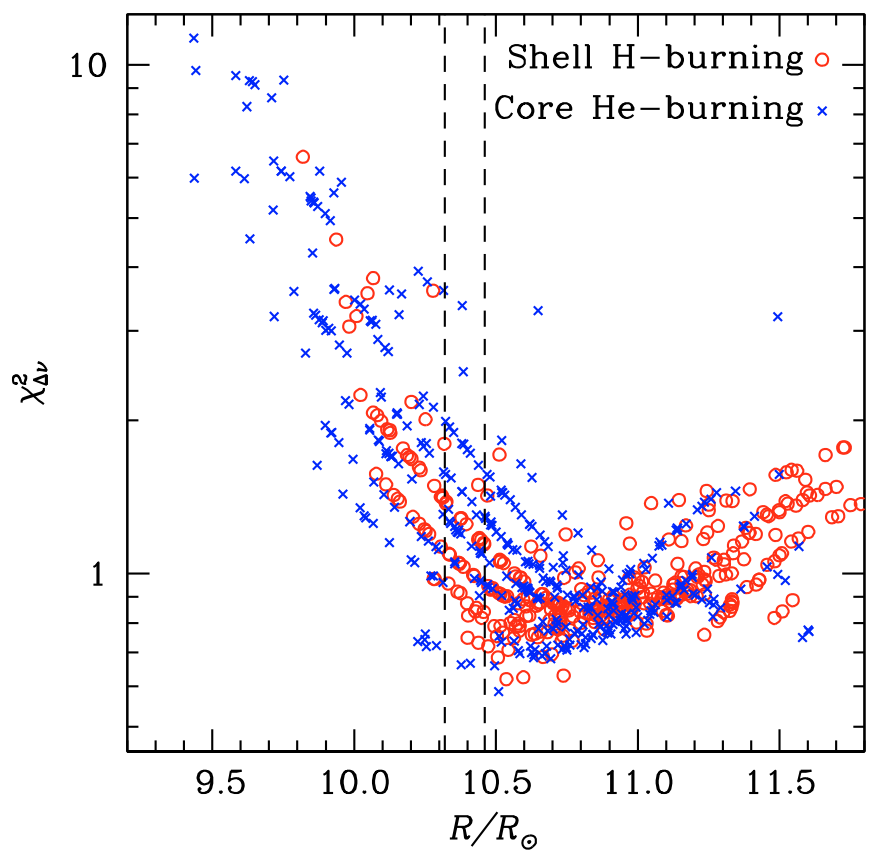

Fig. 10. The $\chi_{\Delta v}^{2}$ values are shown as functions of radius for all stellar models constructed within the $\epsilon$ Oph errorbox. The red circles represent the shell H-burning and the blue crosses represent the core He-burning models. The vertical dashed lines denote the $1 \sigma$ interval for the interferometric radius.

Our interferometric measurements yield a value of the radius of $\epsilon$ Oph that is in close agreement with the radii of our best models obtained through seismic analysis. The interferometric radius was not used as a constraint to choose the best model, but was rather checked a posteriori against the seismic values. The range of possible values of radius of stellar models within the errorbox on the HR diagram for $\epsilon$ Oph is $9 R_{\odot}$ to $12 R_{\odot}$. But the seismic information, specifically the frequencies or the large separation, restricts the radius to a much narrower range. The best-fitting models using large separation comparison have radii of $R \approx 10.5 R_{\odot}$ for both shell $\mathrm{H}$-burning and core He-burning phases. This value is within $2 \sigma$ of the interferometric radius. Even considering all models with $\chi^{2} \leq 1$ constrains the range of radius to $10.4 \leq R / R_{\odot} \leq 11.2$ for frequency comparison, and to $10.2 \leq R / R_{\odot} \leq 11.6$ for large separation comparison (see Fig. 10). This range encompasses the much narrower limit for the radius set by the interferometric measurements. It is remarkable that despite the inherent uncertainties in the modelling of the outer layers of a star, the seismic analysis alone leads to a value of the radius that is in such good agreement with an independent direct estimate of the radius. The radius obtained by Kallinger et al. (2008a) through frequency fitting, $R=10.8 R_{\odot}$, seems to be more removed from the interferometric radius than our seismic value is, although it is difficult to directly compare the two in view of the absence of error bars for the former.

As mentioned above, in the present study the independent estimate of the radius was not used as an additional constraint for choosing the best seismic model. However, since the modelling is in no way influenced by the presence of the radius information, we can check what would be the result if indeed the radius is used as a constraint. We use the $1 \sigma$ interval of the radius to restrict the position of the star on the HR diagram, along with the adopted values of luminosity and effective temperature. This leads to a trapezoidal area on the HR diagram (see Fig. 11) 
Table 4. Stellar parameters for the models with lowest $\chi_{\Delta v}^{2}$ and $\chi_{v}^{2}$ values in either shell H-burning or core He-burning phase that have radii within $\pm 1 \sigma$ of the interferometric radius.

\begin{tabular}{clcccccccccc}
\hline \hline Minimised by & Evolutionary phase & $Y_{0}$ & $Z_{0}$ & $\alpha$ & $M / M_{\odot}$ & $\operatorname{Age}(\mathrm{Myr})$ & $\log T_{\mathrm{eff}}$ & $\log L / L_{\odot}$ & $R / R_{\odot}$ & $\chi_{v}^{2}$ & $\chi_{\Delta v}^{2}$ \\
\hline \multirow{2}{*}{$\chi_{\Delta v}^{2}$ or $\chi_{v}^{2}$} & Shell H-burning & 0.275 & 0.012 & 1.8 & 1.9 & 985 & 3.685 & 1.732 & 10.44 & 3.38 & 0.73 \\
& Core He-burning & 0.270 & 0.013 & 1.8 & 1.8 & 1322 & 3.691 & 1.754 & 10.41 & 3.42 & 0.67 \\
\hline
\end{tabular}

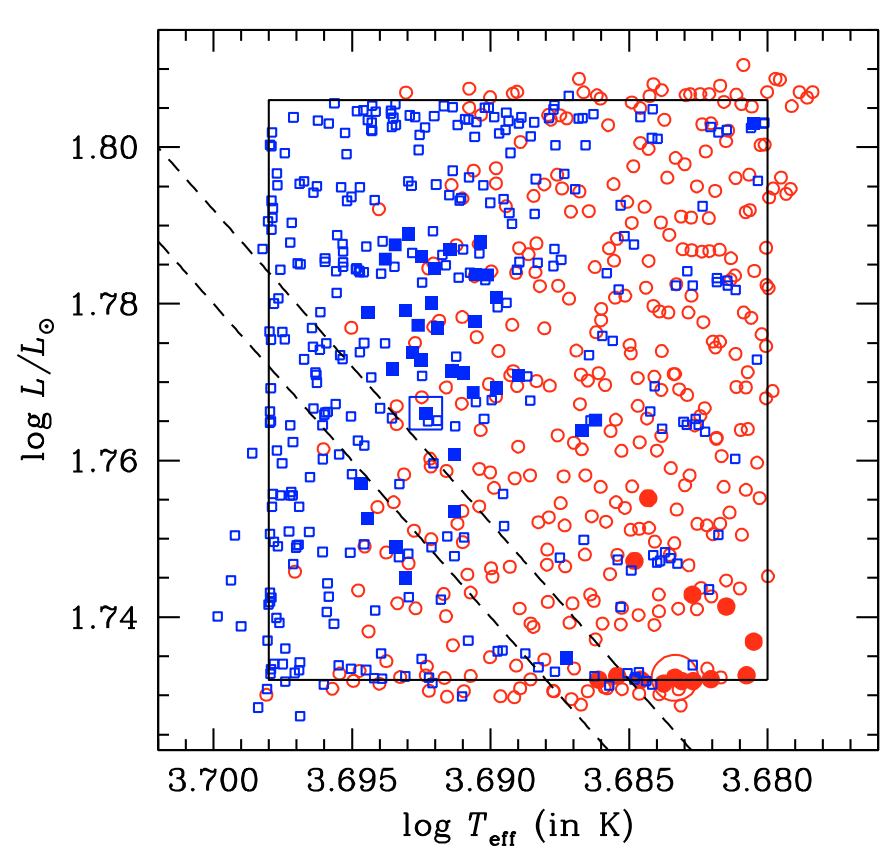

Fig. 11. All the computed stellar models inside and around the $\epsilon$ Oph errorbox on the HR diagram are shown. The red open circles represent the shell H-burning and the blue open squares represent the core He-burning models. The filled symbols represent the models with $\chi_{\Delta,}^{2} \leq 0.8$ for each phase of evolution. The big circle and the big square show the best models in the two phases. The solid line rectangle represents the adopted errorbox of $\epsilon$ Oph. The dashed lines denote the loci of constant radii $R=10.32 R_{\odot}$ and $R=10.46 R_{\odot}$, which are the $\pm 1 \sigma$ bounds for the interferometric radius of $\epsilon$ Oph.

as the errorbox for $\epsilon$ Oph. The parameters of the models inside this smaller errorbox that fit the seismic data best are shown in Table 4. In this case, the minimisations according to frequencies and large separations yield the same best models in either phase of evolution. The minimum values of $\chi_{\Delta v}^{2}$ are marginally higher than in the more general case (cf. Table 1). However, the minimum $\chi_{v}^{2}$ values are significantly higher, indicating that although the observed large separations are quite well matched by these models, the absolute frequency values are somewhat shifted. This is wholly expected since the large separations are strongly influenced by the radius, even if the frequencies themselves may be shifted because of inadequate modelling of the surface effects. In other words, restricting the radius value implies a strong constraint on the large separation, but not necessarily on the absolute frequencies. This is also borne out in the more general case (when the radius constraint is not used) in Fig. 11 where the models with lowest $\chi_{\Delta v}^{2}$ values $(\leq 0.8)$ in either phase of evolution lie in a broad band almost parallel to the interferometric radius band, indicating a constant higher radius value $\left(\sim 10.55 \pm 0.15 R_{\odot}\right)$ common to all of them. The seismic values of the mass and radius of $\epsilon$ Oph yield an average large separation value of $5.35 \pm 0.18 \mu \mathrm{Hz}$ according to the scaling formula of Kjeldsen \& Bedding (1995), which is completely consistent with the current data (Barban et al. 2007).

It is also possible to determine the effective temperature of the star from its measured radius and photometric data. From Fig. 11 itself, it is evident that the intersection of the luminosity limits and the interferometric radius ranges indicates a temperature range of $\log T_{\text {eff }}=3.695 \pm 0.010(4955 \pm 100 \mathrm{~K})$ which is consistent with our adopted range of effective temperature and has a similar uncertainty. Further, a fit of the available photometric data on $\epsilon$ Oph ( $B V J H K$ bands) using tabulated Kurucz models yields $\log T_{\text {eff }}=3.691 \pm 0.002(4912 \pm 25 \mathrm{~K})$ for surface gravity values typical of stars in the relevant zone of the HR diagram. Actually, a change of $0.1 \mathrm{dex}$ in $\log g$ makes a difference of only $1 \mathrm{~K}$ in $T_{\text {eff }}$, while the uncertainty in the measured angular diameter contributes about $4 \mathrm{~K}$ in the error estimate. Again, these value of $T_{\text {eff }}$ are completely contained in the range that we have used from de Ridder et al. (2006). Thus our adopted values of $L$ and $T_{\text {eff }}$ are consistent with the independent measurements of parallax (Hipparcos) and the angular diameter (this paper).

It is evident from this study that the seismic information alone can go a long way in constraining the most important stellar parameters of red giants. Even with a very limited data set, it was possible to obtain a reasonably narrow range of parameters for $\epsilon$ Oph, and the radius estimate from the seismic modelling stands in close agreement with a completely independent interferometric measurement. However, the accuracy of the models can be greatly enhanced by the additional information about the interferometric radius. An independent radius measurement, with a high precision such as provided by interferometry, helps in reducing the size of the errorbox on the HR diagram, making the task of searching for the best model easier. This is the first instance of the coming together of asteroseismology and interferometry for red giant stars, and clearly illustrates the huge potential of this combination in detailed studies of such stars.

Acknowledgements. The authors would like to thank all the CHARA Array and Mount Wilson Observatory day-time and night-time staff for their support. The CHARA Array was constructed with funding from Georgia State University, the National Science Foundation, the W. M. Keck Foundation, and the David and Lucile Packard Foundation. The CHARA Array is operated by Georgia State University with support from the College of Arts and Sciences, from the Research Program Enhancement Fund administered by the Vice President for Research, and from the National Science Foundation under NSF Grant AST 0606958. This work also received the support of PHASE, the high angular resolution partnership between ONERA, Observatoire de Paris, CNRS and University Denis Diderot Paris 7. This research took advantage of the SIMBAD and VIZIER databases at the CDS, Strasbourg (France), and NASA's Astrophysics Data System Bibliographic Services. Part of this work was supported by the Research Fund of K. U. Leuven under grant GOA/2003/04 for AM and CB. S.T.R. acknowledges partial support by NASA grant NNH09AK731. The authors thank Marc-Antoine Dupret and Sarbani Basu for their valuable comments and suggestions.

\section{References}

Alvarez, R., \& Plez, B. 1998, A\&A, 330, 1109

Bahcall, J. N., \& Pinsonneault, M. H. 1992, Rev. Mod. Phys., 64, 885 Barban, C., Matthews, J. M., de Ridder, J., et al. 2007, A\&A, 468, 1033 Böhm-Vitense, E. 1958, ZAp, 46, 108 
Buzasi, D., Catanzarite, J., Laher, R., et al. 2000, ApJ, 532, L133

Cayrel de Strobel, G., Soubiran, C., \& Ralite, N. 2001, A\&A, 373, 159

Christensen-Dalsgaard, J. 2004, Sol. Phys., 220, 137

Claret, A. 2000, A\&A, 363, 1081

Clayton, D. D. 1968, Principles of Stellar Evolution and Nucleosynthesis (New York: McGraw-Hill)

Cohen, M., Walker, R. G., Carter, B., et al. 1999, AJ, 117, 1864

Cole, P. W., \& Deupree, R. G. 1983, ApJ, 269, 676

Coudé du Foresto, V., Ridgway, S., \& Mariotti, J.-M. 1997, A\&AS, 121, 379

Coudé du Foresto, V., Bordé, P., Mérand, A., et al. 2003, Proc. SPIE, 4838, 280

Creevey, O. L., Monteiro, M. J. P. F. G., Metcalfe, T. S., et al. 2007, A\&A, 659, 616

Cunha M. S., Aerts, C., Christensen-Dalsgaard, J., et al. 2007, A\&A Rev., 14, 217

de Ridder, J., Barban, C., Carrier, F., et al. 2006, A\&A, 448, 689

Demarque, P., \& Mengel, J. G. 1971, ApJ, 164, 317

Demarque, P., Woo, J.-H., Kim, Y.-C., et al. 2004, ApJS, 155, 667

Demarque, P., Guenther, D. B., Li, L. H., Mazumdar, A., \& Straka, C. W. 2008, Ap\&SS, 316, 31

Dupret, M.-A., Belkacem, K., Samadi, R., et al. 2009, A\&A, accepted

Dziembowski, W. A., Gough, D. O., Houdek, G., \& Sienkiewicz, R. 2001, MNRAS, 328, 601

ESA 1997, The Hipparcos and Tycho Catalogues, ESA SP-1200

Ferguson, J. W., Alexander, D. R., Allard, F., et al. 2005, ApJ, 623, 585

Frandsen, S., Carrier, F., Aerts, C., et al. 2002, A\&A, 394, L5

Grevesse, N., \& Sauval, A. J. 1998, Space Sci. Rev., 85, 161

Guenther, D. B. 1994, ApJ, 422, 400

Guenther, D. B., Demarque, P., Kim, Y.-C., \& Pinsonneault, M. H. 1992, ApJ, 87,372

Gustafsson, B., Edvardsson, B., Eriksson, K., et al. 2008, A\&A, 486, 951

Hekker, S., Barban, C., \& Kallinger, T. 2008, Commun. Asteroseismol., 157, 319
Iglesias, C. A., \& Rogers, F. J. 1996, ApJ, 464, 943

Itoh, N., Adachi, T., Nakagawa, M., Kohyama, Y., \& Munakata, H. 1989, ApJ, 339, 354

Kallinger, T., Guenther, D. B., Matthews, J. M., et al. 2008a, A\&A, 478, 497

Kallinger, T., Guenther, D. B., Weiss, W. W., et al. 2008b, Commun.

Asteroseismol., 153, 84

Kervella, P., Ségransan, D., \& Coudé du Foresto, V. 2004, A\&A, 425, 1161

Kervella, P., Mérand, A., Pichon, B., et al. 2008, A\&A, 488, 667

Kjeldsen, H., \& Bedding, T. R. 1995, A\&A, 293, 87

Lee, Y.-W., \& Demarque, P. 1990, ApJS, 73, 709

Mérand, A., Ph. D. Thesis 2005, Université Paris 7

Mérand, A., Bordé, P., \& Coudé du Foresto, V. 2005, A\&A, 433, 1155

Mérand, A., Coudé du Foresto, V., Kellerer, A., et al. 2006, Proc. SPIE, 6268, 46

Mocàk, M., Müller, E., Weiss, A., \& Kiforidis, K. 2009, A\&A, 501, 659

Perrin, G. 2003, A\&A, 400, 1173

Piersanti, L., Tornambé, A., \& Castellani, V. 2004, MNRAS, 353, 243

Reimers, D. 1977, A\&A, 57, 395

Rogers, F. J., \& Nayfonov, A. 2002, ApJ, 576, 1064

Schwarzschild, M., \& Härm, R. 1962, ApJ, 136, 158

Straka, C. W., Demarque, P., \& Robinson, F. J. 2007, IAU Symp., 239, 388

Sweigart, A. V. 1987, ApJS, 65, 95

Sweigart, A. V., Greggio, L., \& Renzini, A. 1989, ApJS, 69, 911

ten Brummelaar, T. A., McAlister, H. A., Ridgway, S. T., et al. 2005, ApJ, 628, 453

van Altena, W. F., Lee, J. T., \& Hoffleit, E. D. 1995, The General Catalogue of Trigonometric Stellar Parallaxes, 4th Edn, Yale University Observatory van Leeuwen, F. 2007a, Hipparcos, the New Reduction of the Raw Data, Astrophys. Space Sci. Libr., 350 (Springer)

van Leeuwen, F. 2007b, A\&A, 474, 653

Walker, G., Matthews, J., Kuschnig, R., et al. 2003, PASP, 115, 1023

Yi, S. K., Kim, Y.-C., \& Demarque, P. 2003, ApJS, 144, 259 